\title{
The Association between Number of Remaining Teeth and Maintenance of Successful Aging in Japanese Older People: A 9-Year Longitudinal Study
}

\author{
Fumiya Tanji, ${ }^{1,2}$ Takamasa Komiyama, ${ }^{3}$ Takashi Ohi, ${ }^{3}$ Yoshinori Hattori, ${ }^{3}$ \\ Makoto Watanabe, ${ }^{4}$ Yukai $\mathrm{Lu}^{1}$ and Ichiro Tsuji ${ }^{1}$ \\ ${ }^{1}$ Division of Epidemiology, Department of Health Informatics and Public Health, Tohoku University School of \\ Public Health, Graduate School of Medicine, Sendai, Miyagi, Japan \\ ${ }^{2}$ Faculty of Nursing, Japanese Red Cross Akita College of Nursing, Akita, Akita, Japan \\ ${ }^{3}$ Division of Aging and Geriatric Dentistry, Department Oral Function and Morphology, Tohoku University \\ Graduate School of Dentistry, Sendai, Miyagi, Japan \\ ${ }^{4}$ Institute of Living and Environmental Sciences, Miyagi Gakuin Women's University, Sendai, Miyagi, Japan
}

\begin{abstract}
With population aging, an increasing attention has been paid to quality of life rather than mere longevity. Now, it is urgently needed to clarify predictors of well-being in later life, i.e., "successful aging (SA)." The aim of this study is to investigate whether the number of remaining teeth impacts on maintenance of SA among Japanese older people. The present study was conducted in Tsurugaya district, a suburban area of Sendai, in northern Japan, and included older people aged $\geq 70$ years who had met the criteria for SA at a 2003 baseline survey. At the baseline survey, dentists obtained data for the number of remaining teeth. We obtained information about Long-term Care Insurance certification, including the dates of incident functional disability and death between 2003 and 2012. Data pertaining to health-related quality of life (HRQOL) were collected at the 2003 baseline survey and the 2012 follow-up survey. Maintenance of SA was defined in terms of survival, disability-free status and high HRQOL in both 2003 and 2012. Among 450 participants, $108(24.0 \%)$ were considered to have maintained a state of SA. When participants were classified into three groups according to previous studies, in comparison with participants who retained 0-9 teeth, the multivariate prevalence ratios $(95 \%$ confidence intervals) were $1.39(0.81-2.36)$ for those who retained 10-19 teeth and $1.58(1.002-2.50)$ for those who retained $\geq 20$ teeth $(p$ trend $=0.046)$. The present results suggest that retaining $\geq 20$ teeth is associated with maintenance of SA among Japanese older people.
\end{abstract}

Keywords: Japan; longitudinal study; older people; successful aging; teeth

Tohoku J. Exp. Med., 2020 November, 252 (3), 245-252.

\section{Introduction}

With population aging, an increasing attention has been paid to not only longevity but also well-being in later life, i.e., "successful aging" (Strawbridge et al. 1996; Rowe and Kahn 1997; Milte and McNaughton 2016). A comprehensive definition of successful aging has been proposed by Rowe and Kahn (1997). This concept includes components such as a low probability of disease and disease-related disability, high cognitive and physical functional capacity, and active engagement with life (Rowe and Kahn 1997). In other words, successful agers retain good physical function and psychosocial status (e.g., disability-free status and high health-related quality of life; HRQOL) (Rowe and Kahn 1997; Milte and McNaughton 2016). Previous studies have reported various factors that impact on the achievement of successful aging (to become successful agers), such as lifestyle and social contacts (Strawbridge et al. 1996; Milte and McNaughton 2016). However, no study has clarified the predictors needed for maintaining successful aging for as long as possible.

Dental health is an important determinant of geriatric

Received September 7, 2020; revised and accepted October 21, 2020. Published online November 7, 2020; doi: 10.1620/tjem.252.245. Correspondence: Ichiro Tsuji, Prof., Ph.D., Division of Epidemiology, Department of Health Informatics and Public Health, Tohoku

University School of Public Health, Graduate School of Medicine, 2-1 Seiryo-machi, Aoba-ku, Sendai, Miyagi 980-8575, Japan. e-mail: tsuji1@med.tohoku.ac.jp

(C)2020 Tohoku University Medical Press. This is an open-access article distributed under the terms of the Creative Commons Attribution-NonCommercial-NoDerivatives 4.0 International License (CC-BY-NC-ND 4.0). Anyone may download, reuse, copy, reprint, or distribute the article without modifications or adaptations for non-profit purposes if they cite the original authors and source properly.

https://creativecommons.org/licenses/by-nc-nd/4.0/ 
health. Poor dental health leads to tooth loss through periodontal disease and dental caries. Previous prospective cohort studies have shown that a small number of remaining teeth is associated with an increased risk of mortality and incident functional disability, and a longer life expectancy with functional disability (Holm-Pedersen et al. 2008; Hayasaka et al. 2013; Bando et al. 2017; Matsuyama et al. 2017). Moreover, dental health also affects quality of life among older people. For example, tooth loss adversely affects mastication function, speaking, appearance and social contact, leading to deterioration of oral health-related quality of life (OHRQOL) (Gerritsen et al. 2010). Previous cross-sectional studies have investigated the association between the number of remaining teeth and general HRQOL (Haag et al. 2017a, b). Therefore, we hypothesized that older people who retain a large number of teeth would subsequently maintain successful aging. To our knowledge, no longitudinal study has yet examined the association between the number of remaining teeth and maintenance of successful aging. Such knowledge might lead to a novel strategy for promoting and maintaining successful aging.

The aim of the present prospective cohort study was to investigate the association between the number of remaining teeth and maintenance of successful aging among Japanese older people. To examine this association, we identified successful agers (mean age 74.9 years) in a 2003 survey, and conducted a follow-up survey 9 years later.

\section{Materials and Methods}

\section{Study design and participants}

The Tsurugaya Project was a comprehensive geriatric assessment implemented in 2003 that included medical conditions as well as physical and cognitive function (Kuriyama et al. 2006). We then conducted a follow-up questionnaire study in 2012, which included information about lifestyle habits, HRQOL and other factors. Among 2,925 community-dwelling older people aged 70 years or more and living in the Tsurugaya district, a suburban area of Sendai, in northern Japan, 923 provided written informed consent to participate in the study and agreement to review their Long-term Care Insurance (LTCI) information (Fig. 1).

Of 923 subjects, 409 were excluded because they were not successful agers at the 2003 baseline. We also excluded participants for whom no data about the number of teeth and HRQOL were available $(n=7)$. Subsequently, we followed up 507 participants who had met the criteria for successful aging in the 2003 baseline survey. Of these participants, we later excluded 35 who had moved out of the study area by the time of the follow-up survey in 2012. Then, among participants who had no disability in 2012, we conducted a follow-up questionnaire study at that time, including HRQOL (3-level version of the European Quality of Life-5 Dimensions; EQ-5D-3L), and excluded 22 who

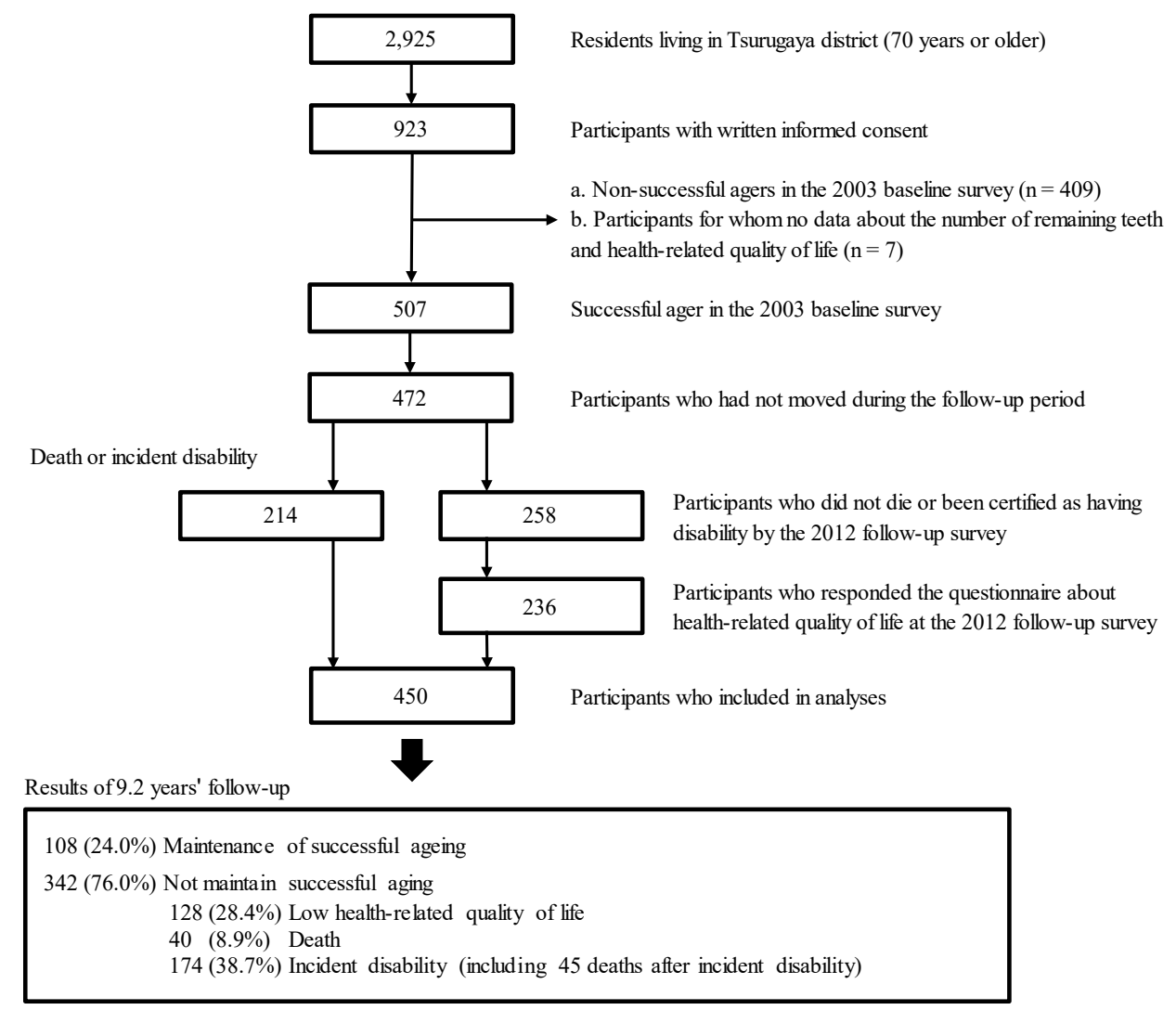

Fig. 1. Flow chart of the study participants: Tsurugaya Project. 
failed to respond to the EQ-5D-3L in 2012. Therefore, for the present study, we analyzed data for 450 participants whose mean age (standard deviation; SD) in 2003 was 74.9 (4.3) years, i.e., the follow-up rate was $88.6 \%$ among 507 eligible participants.

We obtained and used information from the baseline and follow-up surveys, and followed up data on death, emigration and long-term care certification status after confirming that written consent had been obtained from the participants. The Ethics Committee of Tohoku University Graduate School of Medicine approved the protocol of this study (approval number: 2002040; 2010464; 20121113).

\section{Measurements}

Exposure measurement included a count of the number of remaining teeth which was assessed by dentists in the 2003 baseline survey. Any retained roots were excluded from counts of remaining teeth. Similarly to previous studies, we classified the number of remaining teeth into three groups: 0-9, 10-19 and $\geq 20$ (Hayasaka et al. 2013; Tan et al. 2016; Bando et al. 2017).

The questionnaire requested the following information from each participant in the 2003 baseline survey: age at the baseline, sex, medical history (stroke, myocardial infarction, cancer, diabetes mellitus), smoking status, drinking status, physical activity, education level (age at last school graduation). The participants were further tested for cognitive function, and their weight $(\mathrm{kg})$ and height $(\mathrm{m})$ were measured in the 2003 baseline survey. We also obtained blood samples for measurement of the serum albu$\min (\mathrm{g} / \mathrm{dL})$ and C-reactive protein $(\mathrm{CRP})$ levels $(\mathrm{mg} / \mathrm{dL})$ in the 2003 baseline survey.

Physical activity was measured using a self-reported physical activity questionnaire (Fujii et al. 2011). We estimated total daily time expenditure in terms of four domains: occupational activity (including housekeeping and commuting), leisure time activity, sleeping and other activity. The degree of physical activity was calculated as the METs/day score, obtained by summing the number of hours spent on each of the four respective activity domains per day multiplied by the metabolic equivalent, METs $(\mathrm{kcal} / \mathrm{kg} / \mathrm{h})$. In the present study, participants whose total time spent on occupational activity, leisure time activity and sleeping was beyond $24 \mathrm{~h}$ were defined as missing. We classified participants into tertile groups (METs/day $\leq 34.4,34.5-36.6$ and $\geq$ 37.0).

Cognitive function was measured using the MiniMental State Examination (MMSE) (Folstein et al. 1975). Similarly to previous studies, we classified respondents with MMSE scores of $\leq 26$ as having a lower degree of cognitive function (Boban et al. 2012). Body mass index was calculated as measured weight in kilograms divided by measured height in meters squared. Serum albumin levels were measured at a clinical testing laboratory (SRL, Tokyo, Japan). Serum CRP levels were determined using an immunochemical technique on a Behring BN II analyzer
(Dade Behring, Tokyo, Japan).

\section{Study outcome (maintenance of successful aging)}

We defined successful aging as a composite outcome of survival, disability-free status and high HRQOL (EQ-5D-3L score), in accordance with previous studies (Rowe and Kahn 1997; Milte and McNaughton 2016). Therefore, individuals were considered to have maintained successful aging if their status for these parameters was retained in both 2003 and 2012.

HRQOL was measured by the EQ-5D-3L at the baseline survey in June 2003 and at the follow-up survey in September 2012. The questionnaire was self-reported, and included five dimensions: 1) mobility, 2) self-care, 3) usual activities, 4) pain/discomfort, and 5) anxiety/depression (EuroQol Group 1990). Each of the five dimensions was assessed by a single question with three response levels (no problem, some problems, and extreme problems), thus allowing for $3^{5}$ (i.e., 243) possible health combinations overall. The Japanese version of the EQ-5D was developed by Tsuchiya et al (2002). The results were coded and converted to a utility value score ranging from -0.111 to 1.000 . An EQ-5D-3L score of 1.000 represents a state of full health, and we defined this score as "high HRQOL".

In this study, we defined incident functional disability as certification for LTCI in Japan. The LTCI system in Japan employs a nationally uniform standard of functional disability. LTCI is a mandatory form of social insurance aimed at assisting activities of daily living (ADL) of frail older people (Ikegami 1997; Tsutsui and Muramatsu 2005; Ministry of Health, Labour and Welfare 2017). A community-based study has shown that levels of LTCI certification are well correlated with the ability to perform activities of daily living, and with cognitive function (Arai et al. 2003). LTCI certification has already been used in many studies as a measure of incident functional disability in older people (Bando et al. 2017; Tanji et al. 2017; Tomata et al. 2017).

Sendai City Municipal Authority provided annual information about LTCI certification including dates of certification, moving from the study area, and death for the 9.2 years between 1 July 2003 and 30 September 2012.

\section{Statistical analysis}

Baseline characteristics were evaluated using the chisquared test for categorical variables and ANOVA for continuous variables. We conducted longitudinal analyses to evaluate the association between the number of remaining teeth and maintenance of successful aging. We then used the Poisson regression model with robust variance to calculate the adjusted prevalence ratios (PRs) and 95\% confidence intervals (CIs). Respondents who had retained 0-9 teeth were defined as a reference category. For cases where values for a confounding variable were missing, we created a separate category for missing response and included this in the model. The following four models were used to analyze the association between the number of remaining teeth 
and maintenance of successful aging. Model 1 was adjusted by sex and age (70-74, 75-79, $80-84$, or $\geq 85$ years). Model 2 was adjusted by all potential confounding factors, which were sex, age, medical history (stroke, myocardial infarction, cancer, or diabetes mellitus), smoking status (currently, ever, never, or missing), drinking status (currently, ever, never, or missing), physical activity (tertiles or missing), education level $(\leq 17,18-21$, or $\geq 22$ years, or missing) and cognitive function (lower, higher, or missing). For post-hoc analysis, we performed Model 2 among participants who had $<20$ teeth also using denture and those who had $\geq 20$ teeth, after excluding 13 participants who had $<20$ teeth without using denture and one participant who had no data for denture use $(n=436)$. Furthermore, to examine whether inflammatory or nutritional factors could explain the association between the number of remaining teeth and maintenance of successful aging, we adjusted for Model 2 plus the serum CRP level $(<0.3, \geq 0.3 \mathrm{mg} / \mathrm{dl}$, or missing) in Model 3, and did so for Model 2 plus body mass index $(<18.5,18.5$ 24.9 , or $\left.\geq 25.0 \mathrm{~kg} / \mathrm{m}^{2}\right)$ and serum albumin $(<3.8, \geq 3.8 \mathrm{~g} / \mathrm{ml}$, or missing) in Model 4. For post-hoc analysis, to examine whether mental health (using a Japanese version of the 30-item Geriatric Depression Scale score (Wancata and Friedrich 2011)) could explain this association, we adjusted for Model 2 plus mental health.

In addition, we conducted sensitivity analyses by excluding participants who died during the study period: high HRQOL and disability-free status versus those with low HRQOL or disability. This was to check whether the effect of exposure factor on mortality differed from that on functional maintenance.

Statistical analyses were performed using the SAS software package (version 9.4; SAS Institute, Inc., Cary, North Carolina, USA). Poisson regression model were performed using Stata 16.0 software (StataCorp LP). All statistical tests were 2 -sided, and differences at $p<0.05$ were accepted as significant.

\section{Results}

During the 9.2 years of follow-up, the number of participants who were considered to have maintained successful ageing was 108 (24.0\%) (Fig. 1).

Among the 450 participants, the mean age (SD) of the 365 participants who survived up until the 2012 follow-up survey was 82.9 (3.9) years at that point. Among the 85 decedents, those who had $0-9,10-19$ and $\geq 20$ teeth were 39,17 and $29(45.9,20.0$ and $34.1 \%)$, respectively, and the mean age (SD) at baseline and death was 76.7 (5.4) and 81.5 (5.8) years. Baseline characteristics of the participants grouped according to the number of remaining teeth are shown in Table 1. Participants who had retained $\geq 20$ teeth were more likely to be younger, to be current drinkers, to have higher cognitive function and to have a higher serum albumin level $(\mathrm{p}<0.05)$.

The association between the number of remaining teeth and maintenance of successful ageing is shown in
Table 2. In comparison with participants who had 0-9 teeth, PRs $(95 \% \mathrm{CI})$ for maintenance of successful ageing were $1.51(0.88-2.59)$ for those who had 10-19 teeth and 2.21 $(1.42-3.45)$ for those who had $\geq 20$ teeth $(\mathrm{p}$ trend $<0.001)$. Even after adjustment for most confounding factors, Model 2 demonstrated a positive association between the number of remaining teeth and maintenance of successful aging: compared with participants who had 0-9 teeth, multivariate PRs (95\% CI) were $1.39(0.81-2.36)$ for $10-19$ teeth and $1.58(1.002-2.50)$ for $\geq 20$ teeth $(p$ trend $=0.046)$. The multivariate PR per 1-teeth increase $(95 \% \mathrm{CI}, \mathrm{p}$ trend) was 1.02 (0.996-1.03, p trend $=0.105)$ (Table not shown). Among participants who had $<20$ teeth also using denture and those who had $\geq 20$ teeth $(n=436)$, compared with participants who had 0-9 teeth, multivariate PRs (95\% CI) were 1.54 (0.89-2.68) for 10-19 teeth and 1.64 (1.01-2.66) for $\geq 20$ teeth ( $\mathrm{p}$ trend $=0.048)$ (Table not shown). Furthermore, we examined whether inflammation or nutrient factors might explain the association between the number of remaining teeth and maintenance of successful aging. In both Model 3 and Model 4, the number of remaining teeth was positively associated with maintenance of successful aging even after adding inflammation or nutrient factors to the multivariate model (Model 2). After adjustment for Model 2 plus mental health, compared with participants who had 0-9 teeth, multivariate PRs $(95 \% \mathrm{CI})$ were $1.41(0.83-2.40)$ for $10-19$ teeth and $1.51(0.95-2.39)$ for $\geq$ 20 teeth $(\mathrm{p}$ trend $=0.080)$ (Table not shown).

Table 3 shows the results of sensitivity analyses excluding 85 individuals who died during the study period: high HRQOL and disability-free status versus low HRQOL or disability. Although this association was marginally significant after adjustment for most confounding factors in Model 2, the result remained unchanged from the former one (Table 2): compared with participants who had 0-9 teeth, multivariate PRs $(95 \% \mathrm{CI})$ were $1.28(0.75-2.16)$ for $10-19$ teeth and $1.49(0.94-2.34)$ for $\geq 20$ teeth ( $\mathrm{p}$ trend $=$ 0.080). The results obtained in Models 3 and 4 remained unchanged.

\section{Discussion}

The present longitudinal study was designed to clarify predictors of the maintenance of successful aging among Japanese older people in a prospective cohort study, and we examined the association between the number of remaining teeth and maintenance of successful aging. We conducted a follow-up survey 9 years after obtaining data for "successful agers" whose mean age had been 74.9 years at the time of the baseline survey. The results suggested that the proportion of individuals who maintained successful aging was significantly higher among those who had 20 teeth or more.

For the present study, we adopted a composite outcome of survival, disability-free status and high HRQOL to indicate maintenance of successful aging. As the effect of exposure factor on mortality differed from that on functional maintenance, we conducted sensitivity analyses by 
Table 1. Baseline characteristics of the study participants according to number of remaining teeth $(\mathrm{n}=450)$.

\begin{tabular}{|c|c|c|c|c|}
\hline & \multicolumn{4}{|c|}{ Number of remaining teeth } \\
\hline & $0-9$ & $10-19$ & $\geq 20$ & $\mathrm{p}$ values \\
\hline No. of subjects & 145 & 105 & 200 & \\
\hline Age (years) $($ mean $\pm \mathrm{SD})$ & $76.4 \pm 4.9$ & $75.4 \pm 4.4$ & $73.5 \pm 3.2$ & $<0.001$ \\
\hline \multicolumn{5}{|l|}{$\operatorname{Sex}(\%)$} \\
\hline Men & 51.0 & 56.2 & 62.0 & 0.124 \\
\hline Women & 49.0 & 43.8 & 38.0 & \\
\hline \multicolumn{5}{|l|}{ Medical history (\%) } \\
\hline Stroke & 4.1 & 4.8 & 0.5 & 0.037 \\
\hline Myocardial infarction & 6.2 & 6.7 & 9.0 & 0.579 \\
\hline Cancer & 8.3 & 7.6 & 7.5 & 0.963 \\
\hline Diabetes mellitus & 15.2 & 10.5 & 14.5 & 0.525 \\
\hline \multicolumn{5}{|l|}{ Smoking status (\%) } \\
\hline Never & 53.1 & 46.7 & 51.0 & 0.193 \\
\hline Former & 30.3 & 34.3 & 39.0 & \\
\hline Current & 15.9 & 15.2 & 9.0 & \\
\hline Missing & 0.7 & 3.8 & 1.0 & \\
\hline \multicolumn{5}{|l|}{ Drinking status (\%) } \\
\hline Never & 42.8 & 32.4 & 29.0 & 0.007 \\
\hline Former & 12.4 & 8.6 & 7.5 & \\
\hline Current & 37.9 & 50.5 & 58.5 & \\
\hline Missing & 6.9 & 8.5 & 5.0 & \\
\hline \multicolumn{5}{|c|}{ Metabolic equivalent/day score (physical activity) (\%) } \\
\hline Higher $(\geq 37.0)$ & 28.3 & 21.9 & 29.0 & 0.793 \\
\hline Moderate (34.5-36.6) & 26.9 & 29.5 & 26.0 & \\
\hline Lower $(\leq 34.4)$ & 29.0 & 26.7 & 26.5 & \\
\hline Missing & 15.8 & 21.9 & 18.5 & \\
\hline \multicolumn{5}{|c|}{ Education level (age at last school graduation) (\%) } \\
\hline$\geq 22$ years & 15.9 & 16.2 & 23.5 & 0.066 \\
\hline $18-21$ years & 47.6 & 57.1 & 49.5 & \\
\hline$\leq 17$ years & 35.8 & 24.8 & 24.5 & \\
\hline Missing & 0.7 & 1.9 & 2.5 & \\
\hline \multicolumn{5}{|c|}{ Mini-Mental State Examination score (cognitive function) (\%) } \\
\hline Higher $(\geq 27)$ & 81.4 & 84.8 & 90.5 & 0.041 \\
\hline Lower $(\leq 26)$ & 17.2 & 15.2 & 8.5 & \\
\hline Missing & 1.4 & 0.0 & 1.0 & \\
\hline \multicolumn{5}{|l|}{ Body mass index $\left(\mathrm{kg} / \mathrm{m}^{2}\right)(\%)$} \\
\hline$\geq 25.0$ & 37.9 & 33.3 & 41.5 & 0.075 \\
\hline $18.5-24.9$ & 55.2 & 60.0 & 57.0 & \\
\hline$\leq 18.4$ & 6.9 & 6.7 & 1.5 & \\
\hline Missing & 0.0 & 0.0 & 0.0 & \\
\hline Serum albumin $(\mathrm{g} / \mathrm{dL} /$ day $)($ mean $\pm \mathrm{SD})$ & $4.1 \pm 0.2$ & $4.2 \pm 0.2$ & $4.2 \pm 0.2$ & $<0.001$ \\
\hline C-reactive protein $(\mathrm{mg} / \mathrm{dL})($ mean $\pm \mathrm{SD})$ & $0.1 \pm 0.3$ & $0.1 \pm 0.2$ & $0.1 \pm 0.1$ & 0.276 \\
\hline
\end{tabular}

We used $\chi 2$ test for variables of proportion and one-factor ANOVA for continuous variables (missing value excluded). $\mathrm{SD}$, standard deviation.

excluding individuals who died during the follow-up period. However, the results were consistent with our main findings.

Caries, periodontal disease and oral inflammation are partly responsible for tooth loss in older people, and these are associated with an increased risk of cerebrovascular disease (Joshipura et al. 2003; Grau et al. 2004). This pathway may result in functional disability. Additionally, tooth loss may affect dietary intake and lead to deterioration of nutritional status (Sheiham et al. 2001). Previous studies have 
Table 2. Association between the number of remaining teeth and maintenance of successful aging $(\mathrm{n}=450)$.

\begin{tabular}{|c|c|c|c|c|}
\hline & \multicolumn{3}{|c|}{ Number of remaining teeth } & \multirow{3}{*}{$\mathrm{p}$ trend } \\
\hline & \multirow[t]{2}{*}{$0-9$} & $10-19$ & $\geq 20$ & \\
\hline & & PR $(95 \% \mathrm{CI})$ & PR $(95 \% \mathrm{CI})$ & \\
\hline Number of subjects & 145 & 105 & 200 & \\
\hline Number of maintenances of successful aging & 21 & 23 & 64 & \\
\hline Crude model & 1.00 (Ref.) & $1.51(0.88-2.59)$ & $2.21(1.42-3.45)$ & $<0.001$ \\
\hline Model $1^{*}$ & 1.00 (Ref.) & $1.44(0.86-2.43)$ & $1.74(1.12-2.72)$ & 0.012 \\
\hline Model $2^{\dagger}$ & 1.00 (Ref.) & $1.39(0.81-2.36)$ & $1.58(1.002-2.50)$ & 0.046 \\
\hline Model $3^{\ddagger}$ & 1.00 (Ref.) & $1.37(0.80-2.33)$ & $1.59(1.002-2.52)$ & 0.045 \\
\hline Model $4^{\S}$ & 1.00 (Ref.) & $1.40(0.80-2.44)$ & $1.61(0.996-2.60)$ & 0.047 \\
\hline
\end{tabular}

PR, prevalence ratio; CI, confidence interval; Ref., referent values.

*Adjustment items : sex, age (70-74, 75-79, 80-84, or $\geq 85$ years).

${ }^{\dagger}$ Adjustment items : sex, age (70-74, 75-79, 80-84, or $\geq 85$ years), medical history (stroke, cancer, myocardial infarction, or diabetes mellitus), smoking status (currently, ever, never, or missing), drinking status (currently, ever, never, or missing), metabolic equivalent/day score (tertiles or missing), education level ( $\leq 17,18-21$, or $\geq 22$ years, or missing), and Mini-Mental State Examination score $(\leq 26, \geq 27$, or missing).

\#Adjustment items: model 2 plus C-reactive protein $(<0.3, \geq 0.3 \mathrm{mg} / \mathrm{dl}$, or missing).

$\S$ Adjustment items: model 2 plus body mass index $\left(<18.5,18.5-24.9\right.$, or $\left.\geq 25.0 \mathrm{~kg} / \mathrm{m}^{2}\right)$ and albumin $(<3.8, \geq 3.8 \mathrm{~g} / \mathrm{ml}$, or missing).

Table 3. Sensitivity analyses after excluding individuals who died during the follow-up period $(\mathrm{n}=365)$.

\begin{tabular}{|c|c|c|c|c|}
\hline & \multicolumn{3}{|c|}{ Number of remaining teeth } & \multirow{3}{*}{$\mathrm{p}$ trend } \\
\hline & \multirow{2}{*}{$0-9$} & $10-19$ & $\geq 20$ & \\
\hline & & PR $(95 \% \mathrm{CI})$ & $\mathrm{PR}(95 \% \mathrm{CI})$ & \\
\hline Number of subjects & 106 & 88 & 171 & \\
\hline Number of high HRQOL and disability-free status & 21 & 23 & 64 & \\
\hline Crude model & 1.00 (Ref.) & $1.32(0.78-2.22)$ & $1.89(1.23-2.90)$ & 0.002 \\
\hline Model $1^{*}$ & 1.00 (Ref.) & $1.23(0.75-2.04)$ & $1.51(0.99-2.33)$ & 0.048 \\
\hline Model $2^{\dagger}$ & 1.00 (Ref.) & $1.28(0.75-2.16)$ & $1.49(0.94-2.34)$ & 0.080 \\
\hline Model $3^{\ddagger}$ & 1.00 (Ref.) & $1.25(0.74-2.11)$ & $1.46(0.93-2.31)$ & 0.091 \\
\hline Model $4^{\S}$ & 1.00 (Ref.) & $1.29(0.75-2.23)$ & $1.53(0.95-2.49)$ & 0.073 \\
\hline
\end{tabular}

PR, prevalence ratio; CI, confidence interval; Ref., referent values; HRQOL, health related quality of life.

*Adjustment items : sex, age (70-74, 75-79, 80-84, or $\geq 85$ years).

${ }^{\dagger}$ Adjustment items : sex, age (70-74, 75-79, 80-84, or $\geq 85$ years), medical history (stroke, cancer, myocardial infarction, or diabetes mellitus), smoking status (currently, ever, never, or missing), drinking status (currently, ever, never, or missing), metabolic equivalent/day score (tertiles or missing), education level ( $\leq 17,18-21$, or $\geq 22$ years, or missing), and Mini-Mental State Examination score $(\leq 26, \geq 27$, or missing).

Adjustment items: model 2 plus C-reactive protein $(<0.3, \geq 0.3 \mathrm{mg} / \mathrm{dl}$, or missing).

$\S$ Adjustment items: model 2 plus body mass index $\left(<18.5,18.5-24.9\right.$, or $\left.\geq 25.0 \mathrm{~kg} / \mathrm{m}^{2}\right)$ and albumin $(<3.8, \geq 3.8 \mathrm{~g} / \mathrm{ml}$, or missing $)$.

shown that poor nutritional status is associated with a higher risk of functional disability and mortality (Shakersain et al. 2016; Zhang et al. 2016). In the present study, we considered the serum CRP level to be an inflammatory factor, and serum albumin and body mass index to be nutritional factors. Additionally, because previous studies have reported that the negative effect of tooth loss on mortality and incident disability (Hayasaka et al. 2013; Bando et al. 2017) was attenuated by using denture, we performed the post-hoc analysis among participants who had $<$ 20 teeth also using denture and those who had $\geq 20$ teeth (n
$=436$ ). However, these results remained unchanged. Their impact on the association between the number of remaining teeth and maintenance of successful aging might be small, as 450 participants included in analysis were successful agers (i.e., healthier) at the baseline.

Mental health may be one of several important issues that can explain the association between the number of remaining teeth and maintenance of successful aging. Posthoc analysis showed that mental health decreased the multivariate PR by $12.0 \%$, which represented a greater impact than inflammatory and nutritional factors. The number of 
remaining teeth is also associated with OHRQOL (Gerritsen et al. 2010; Tan et al. 2016), which has a large impact on mental health because it assesses not only mastication, but also speaking, appearance and social contact (Hassel et al. 2011; Ikebe et al. 2012; Tan et al. 2016). Therefore, successful agers who retain more teeth might be better capable of subsequently maintaining successful aging.

The present study had some methodological strengths. First, it is the first study to have examined the association between the number of remaining teeth and maintenance of successful aging using a longitudinal design. Second, the number of teeth was assessed by dentists, and was not selfreported. Additionally, we used validated measurements functional disability (LTCI certification) and HRQOL (EQ-5D-3L) - which have been used in many previous studies. Third, we considered many confounding factors including lifestyle factors and cognitive function, as well as mediating factors, i.e., inflammatory and nutritional factors.

This study also had some limitations. First, we did not consider all potential confounding factors, such as socioeconomic status, that might have impacted on the association between the number of remaining teeth and maintenance of successful aging. We were unable to obtain information on household income and subjective economic status, although we did consider the education levels of the participants. Second, we obtained HRQOL data at only two time points, i.e., at the baseline and at the follow-up survey, although we did obtain information on the dates of death, moving from the study area, and incident functional disability. Third, the population sample size used in the present study may have been somewhat small.

In conclusion, the present prospective cohort study has shown that retaining 20 teeth or more was associated with maintenance of successful aging among Japanese older people. Further longitudinal studies should be conducted to confirm the impact of the number of remaining teeth on maintenance of successful aging.

\section{Acknowledgments}

This work was supported by Grant-in-Aid for Scientific Research (B) (JSPS KAKENHI Grant Number JP17H04130) from Japan Society for the Promotion of Science.

We would like to thank Yoshiko Nakata for her technical assistance.

\section{Conflict of Interest}

The authors declare no conflict of interest.

\section{References}

Arai, Y., Zarit, S.H., Kumamoto, K. \& Takeda, A. (2003) Are there inequities in the assessment of dementia under Japan's LTC insurance system? Int. J. Geriatr. Psychiatry, 18, 346-352.

Bando, S., Tomata, Y., Aida, J., Sugiyama, K., Sugawara, Y. \& Tsuji, I. (2017) Impact of oral self-care on incident functional disability in elderly Japanese: the Ohsaki Cohort 2006 study. BMJ Open, 7, e017946.
Boban, M., Malojčić, B., Mimica, N., Vuković, S., Zrilić, I., Hof, P.R. \& Simić, G. (2012) The reliability and validity of the mini-mental state examination in the elderly Croatian population. Dement. Geriatr. Cogn. Disord., 33, 385-392.

EuroQol Group (1990) EuroQol: a new facility for the measurement of health-related quality of life. Health Policy, 16, 199-208.

Folstein, M.F., Folstein, S.E. \& McHugh, P.R. (1975) "Minimental state". A practical method for grading the cognitive state of patients for the clinician. J. Psychiatr. Res., 12, 189-198.

Fujii, H., Yamamoto, S., Takeda-Imai, F., Inoue, M., Tsugane, S., Kadowaki, T. \& Noda, M. (2011) Validity and applicability of a simple questionnaire for the estimation of total and domainspecific physical activity. Diabetology International, 2, 47-54.

Gerritsen, A.E., Allen, P.F., Witter, D.J., Bronkhorst, E.M. \& Creugers, N.H. (2010) Tooth loss and oral health-related quality of life: a systematic review and meta-analysis. Health Qual. Life Outcomes, 8, 126.

Grau, A.J., Becher, H., Ziegler, C.M., Lichy, C., Buggle, F., Kaiser, C., Lutz, R., Bültmann, S., Preusch, M. \& Dörfer, C.E. (2004) Periodontal disease as a risk factor for ischemic stroke. Stroke, 35, 496-501.

Haag, D.G., Peres, K.G., Balasubramanian, M. \& Brennan, D.S. (2017a) Oral conditions and health-related quality of life: a systematic review. J. Dent. Res., 96, 864-874.

Haag, D.G., Peres, K.G. \& Brennan, D.S. (2017b) Tooth loss and general quality of life in dentate adults from Southern Brazil. Qual. Life Res., 26, 2647-2657.

Hassel, A.J., Danner, D., Schmitt, M., Nitschke, I., Rammelsberg, P. \& Wahl, H.W. (2011) Oral health-related quality of life is linked with subjective well-being and depression in early old age. Clin. Oral Investig., 15, 691-697.

Hayasaka, K., Tomata, Y., Aida, J., Watanabe, T., Kakizaki, M. \& Tsuji, I. (2013) Tooth loss and mortality in elderly Japanese adults: effect of oral care. J. Am. Geriatr. Soc., 61, 815-820.

Holm-Pedersen, P., Schultz-Larsen, K., Christiansen, N. \& Avlund, K. (2008) Tooth loss and subsequent disability and mortality in old age. J. Am. Geriatr. Soc., 56, 429-435.

Ikebe, K., Hazeyama, T., Enoki, K., Murai, S., Okada, T., Kagawa, R., Matsuda, K. \& Maeda, Y. (2012) Comparison of GOHAI and OHIP-14 measures in relation to objective values of oral function in elderly Japanese. Community Dent. Oral Epidemiol., 40, 406-414.

Ikegami, N. (1997) Public long-term care insurance in Japan. JAMA, 278, 1310-1314.

Joshipura, K.J., Hung, H.C., Rimm, E.B., Willett, W.C. \& Ascherio, A. (2003) Periodontal disease, tooth loss, and incidence of ischemic stroke. Stroke, 34, 47-52.

Kuriyama, S., Koizumi, Y., Matsuda-Ohmori, K., Seki, T., Shimazu, T., Hozawa, A., Awata, S. \& Tsuji, I. (2006) Obesity and depressive symptoms in elderly Japanese: the Tsurugaya Project. J. Psychosom. Res., 60, 229-235.

Matsuyama, Y., Aida, J., Watt, R.G., Tsuboya, T., Koyama, S., Sato, Y., Kondo, K. \& Osaka, K. (2017) Dental status and compression of life expectancy with disability. J. Dent. Res., 96, 1006-1013.

Milte, C.M. \& McNaughton, S.A. (2016) Dietary patterns and successful ageing: a systematic review. Eur. J. Nutr., 55, 423-450

Ministry of Health, Labour and Welfare (2017) Long-term care, health and welfare services for the elderly.

http://www.mhlw.go.jp/english/policy/care-welfare/carewelfare-elderly/

[Accessed: September 2, 2020].

Rowe, J.W. \& Kahn, R.L. (1997) Successful aging. Gerontologist, 37, 433-440.

Shakersain, B., Santoni, G., Faxén-Irving, G., Rizzuto, D., Fratiglioni, L. \& Xu, W. (2016) Nutritional status and survival 
among old adults: an 11-year population-based longitudinal study. Eur. J. Clin. Nutr., 70, 320-325.

Sheiham, A., Steele, J.G., Marcenes, W., Lowe, C., Finch, S., Bates, C.J., Prentice, A. \& Walls, A.W. (2001) The relationship among dental status, nutrient intake, and nutritional status in older people. J. Dent. Res., 80, 408-413.

Strawbridge, W.J., Cohen, R.D., Shema, S.J. \& Kaplan, G.A. (1996) Successful aging: predictors and associated activities. Am. J. Epidemiol., 144, 135-141.

Tan, H., Peres, K.G. \& Peres, M.A. (2016) Retention of teeth and oral health-related quality of life. J. Dent. Res., 95, 13501357.

Tanji, F., Sugawara, Y., Tomata, Y., Watanabe, T., Sugiyama, K., Kaiho, Y., Tomita, H. \& Tsuji, I. (2017) Psychological distress and the incident risk of functional disability in elderly survivors after the Great East Japan Earthquake. J. Affect. Disord., 221, 145-150.

Tomata, Y., Watanabe, T., Sugiyama, K., Zhang, S., Sugawara, Y. \& Tsuji, I. (2017) Effects of a community-based program for oral health and nutrition on cost-effectiveness by preventing disability in Japanese frail elderly: a quasi-experimental study using propensity score matching. J. Am. Med. Dir. Assoc., 18, 678-685.

Tsuchiya, A., Ikeda, S., Ikegami, N., Nishimura, S., Sakai, I., Fukuda, T., Hamashima, C., Hisashige, A. \& Tamura, M. (2002) Estimating an EQ-5D population value set: the case of Japan. Health Econ., 11, 341-353.

Tsutsui, T. \& Muramatsu, N. (2005) Care-needs certification in the long-term care insurance system of Japan. J. Am. Geriatr. Soc., 53, 522-527.

Wancata, J. \& Friedrich, F. (2011) Depression: a diagnosis aptly used? Psychiatr. Danub., 23, 406-411.

Zhang, S., Tomata, Y., Sugiyama, K., Kaiho, Y., Honkura, K., Watanabe, T., Tanji, F., Sugawara, Y. \& Tsuji, I. (2016) Body mass index and the risk of incident functional disability in elderly Japanese: the OHSAKI Cohort 2006 Study. Medicine (Baltimore), 95, e4452. 